\title{
TAFONOMÍA DE LOS TRONCOS SILICIFICADOS ALBIENSES DEL YACIMIENTO DE "EL BARRANQUILLO" (CASTELLOTE, PROVINCIA DE TERUEL, ESPAÑA)
}

\author{
Pablo MUÑOZ BARRAGÁN ${ }^{1}$, José Bienvenido DIEZ ${ }^{1,2}$, \\ Javier FERRER ${ }^{l}$ y Ana Rosa SORIA ${ }^{3}$
}

\author{
1 Área de Paleontología. Departamento de Geología. Universidad de Zaragoza. 50009 Zaragoza. \\ 2 Laboratoire de Paléobotanique et Paléoécologie. Université Paris Vl, 12 rue Cuvier. 75005. \\ France. \\ 3 Área de Estratigrafía. Departamento de Geología. Universidad de Zaragoza. 50009 Zaragoza.
}

\begin{abstract}
Muñoz Barragán, P., Diez J.B., Ferrer, J. y Soria, A.R. 1999. Tafonomía de los troncos silicificados albienses del yaciemiento de "El Barranquillo" (Castellote, provincia de Teruel, España). [Taphonomy of Albian silicified trunks from the locality "El Barranquillo" (Castellote, Teruel, Spain]. Revista Española de Paleontología, 14(2), 165-172. ISSN 0213-6937.
\end{abstract}

\begin{abstract}
Lower Cretaceous fossil trunks from the locality "El Barranquillo" near Castellote are described. From the many trunks four have been excavated. The taphonomic history of the site has been reconstructed, based on the lithofacies, position and conservation of the fossils and petrographic data.

The trunks are supposedly derived from an upstream area, probably uprooted by an episodic (non-seasonal) flood and rapidly buried in the lower course of the stream. Diagenetic processes of carbonization and silicification started soon after burial, leading to the excellent preservation of the trunks.
\end{abstract}

Keywords: Taphonomy, fossil trunks, gimnosperms, Albian, Iberian Range.

\section{RESUMEN}

Se estudia el yacimiento de troncos fósiles de "El Barranquillo" (Cretácico Inferior) situado en Castellote (Teruel). Está formado por un número indeterminado de troncos, de los cuales cuatro han sido excavados. A partir de las observaciones de la facies litológica, de la posición y conservación de los fósiles y de estudios petrográficos se ha podido reconstruir la historia tafonómica del yacimiento.

Los troncos fueron arrancados por una avenida esporádica, no estacional, y arrastrados aguas abajo del río donde se enterraron rápidamente. A partir de ese momento se produjeron los procesos de carbonificación y silicificación típicamente diagenéticos.

Palabras clave: Tafonomía, troncos fósiles, gimnospermas, Albiense, Cordillera Ibérica.

\section{INTRODUCCIÓN}

El yacimiento de troncos fósiles de "El Barranquillo" se sitúa al norte del término municipal de Castellote (provincia de Teruel, Fig. 1), en las cercanías del Pilón de San Pedro.

El descubrimiento y tratamiento del mismo es un claro ejemplo de buena colaboración entre una sociedad cultural local sensibilizada por la conservación de su patrimonio y la Universidad, en nuestro caso el Grupo de Estudios Masinos y el Área de Paleontología de la Universidad de Zaragoza, respectivamente.

El yacimiento fue descubierto por el director del
Museo de Mas de las Matas, Don Antonio Martín Costea. Hasta la década de los años 90, Antonio no dio a conocer este hallazgo por temor a que fuera expoliado. En el año 1993, mostró el yacimiento a un equipo del Museo Paleontológico de la Universidad de Zaragoza, liderado por el catedrático de Paleontología Dr. Eladio Liñán. En esta visita, se pudo contrastar el doble interés del yacimiento, por una parte el científico y por otra el sociocultural.

La excavación paleontológica se inició en el año 1995, con la inestimable colaboración del G.E.M.A. (Grupo de Estudios Masinos), que aportó la maquinaria y el personal necesario para la realización de la misma. 


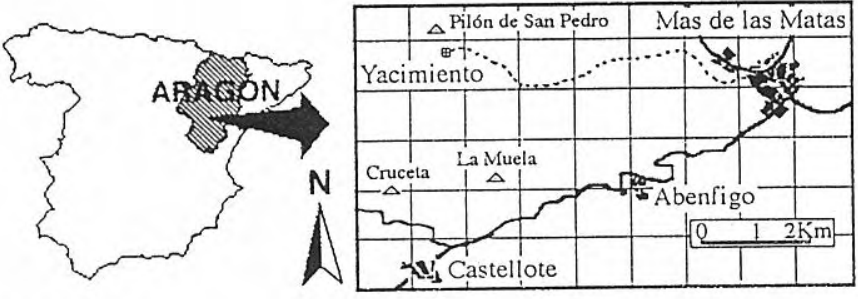

Figura 1. Situación geográfica del yacimiento.

Además de la actuación científica, se ha realizado un acondicionamiento del yacimiento, acción que integra tanto la restauración y tratamientos de conservación de los ejemplares, como la habilitación de las vías de acceso que facilitan la visita de este punto de interés paleobotánico.

Estratigráficamente este yacimiento se localiza dentro de la Formación Utrillas, que se sitúa entre las unidades marinas del Cretácico Inferior (Facies Urgon) y las calizas del Cenomaniense (Cretácico Superior) que coronan el Pilón de San Pedro y montes adyacentes (Fig. 2). Geológicamente se ubica dentro del Arco BeceitePortalrubio (Canerot, 1974) en la Zona de Enlace entre la Cordillera Ibérica y las Catalánides (Guimerà, 1983,1988).

Hasta ahora, la única cita sobre estudios paleobotánicos de troncos silicificados en el Cretácico Inferior de Aragón es el trabajo de Lemoigne y Marin (1972). Estos autores centraron su estudio únicamente en la identificación taxonómica de algunos fragmentos recogidos en los yacimientos de Oliete, Seno y Ladruñán, localidades situadas en las cercanías de Castellote, en la misma provincia de Teruel. Sin embargo, en ninguno de los tres yacimientos los autores citados realizaron un estudio tafonómico como el que presentamos en este trabajo.

\section{ESTRATIGRAFÍA E INTERPRETACIÓN SEDIMENTOLÓGICA}

Como se ha comentado anteriormente, el nivel fosilífero se localiza en la Formación Utrillas (Aguilar et al., 1971). Litológicamente esta unidad está formada por arenas y areniscas alternando con lutitas. Las areniscas, de grano fino a grueso, se presentan en estratos de geometría tabular, lenticular y canaliforme de potencia métrica. Son frecuentes las cicatrices internas y las bases erosivas. Se reconoce estratificación cruzada en surco y planar, laminación paralela o de bajo ángulo y laminación cruzada por migración de ripples de corriente y, en ocasiones, de oscilación. También son frecuentes los cantos blandos, constituyendo depósitos residuales. Las lutitas son normalmente versicolores, en niveles de potencia decimétrica a decamétrica. Incluyen nódulos de hierro, abundantes restos vegetales silicificados y frecuentes niveles de potencia centimétrica y decimétrica de areniscas de grano fino.

En el yacimiento de "El Barranquillo", las arenas son blancas, de grano fino, dispuestas en cuerpos de geometría tabular y potencia métrica. Se reconoce en ellos estratificación cruzada en surco en sets de potencia decimétrica, cuyas láminas presentan una dirección media 060, 20 SE. Se observan costras ferruginosas y estructuras formadas por la migración diagenética de óxidos de hierro. Las lutitas son versicolores, de potencia decamétrica, y presentan abundantes nódulos de hierro. Tanto en las lutitas como en las arenas aparecen incluidos restos de xilema silicificados, cuyo tamaño varía desde pocos centímetros hasta 5 metros.

En esta zona, la Formación Utrillas se dispone sobre las calizas arenosas de la Formación Chert, de edad Aptiense inferior, mediante contacto discordante. Este límite lleva asociada una laguna estratigráfica que abarca el intervalo Aptiense inferior-Albiense inferior. A techo limita con la Formación Mosqueruela, que en esta cuenca ha sido datada como Albiense superior-Cenomaniense inferior (Pardo et al., 1991). Este límite se sitúa unos 40 metros por encima del yacimiento, y se observa nítidamente en el cercano cerro del Pilón de San Pedro. Cuando los planos de estratificación de la Formación Utrillas son visibles en las proximidades del yacimiento, se observa que la serie se dispone subhorizontalmente.

La Formación Utrillas responde, en su conjunto, a un esquema transgresivo, que se inicia con una sedimentación de carácter fluvial que evoluciona hacia una sedimentación detrítico-calcárea típica de medios transicionales (Pardo, 1979), siendo la sedimentación fluvial la responsable de la mayor parte de las sucesiones que integran esta unidad.

A diferencia de gran parte de las Cuencas del Bajo Aragón Turolense (cuencas de Aliaga-Utrillas y EstercuelAriño), en la cuenca del Maestrazgo la sedimentación fluvial se caracteriza por potentes secuencias que, por su evolución estructural y por la geometría de las facies, son referibles a depósitos de ríos meandriformes. En esta cuenca, la evolución hacia la distalidad de la red fluvial se manifiesta en la menor granulometría de los depósitos y en el importante desarrollo de facies de acreción vertical hacia el SE.

Pardo et al. (1991) reconocen 6 secuencias tipo en la Fm. Utrillas. La asociación de facies observada en el yacimiento de "El Barranquillo", compuesta por un término arenoso que

\section{Lámina I}

Aspecto general de la actuación realizada en el yacimiento de "El Barranquillo". Cada división de la barra, en las figuras 2 a 4 , equivale a $10 \mathrm{~cm}$.

1 Vista del tronco silicificado de mayor tamaño.

2 Tronco silicificado de menor tamaño extraído del yacimiento.

3 Tronco desgajado por su parte central en el cual se aprecia la conservación de los vasos leñosos.

4 Aspecto de campo de uno de los ejemplares visibles en el yacimiento. 
Lámina I

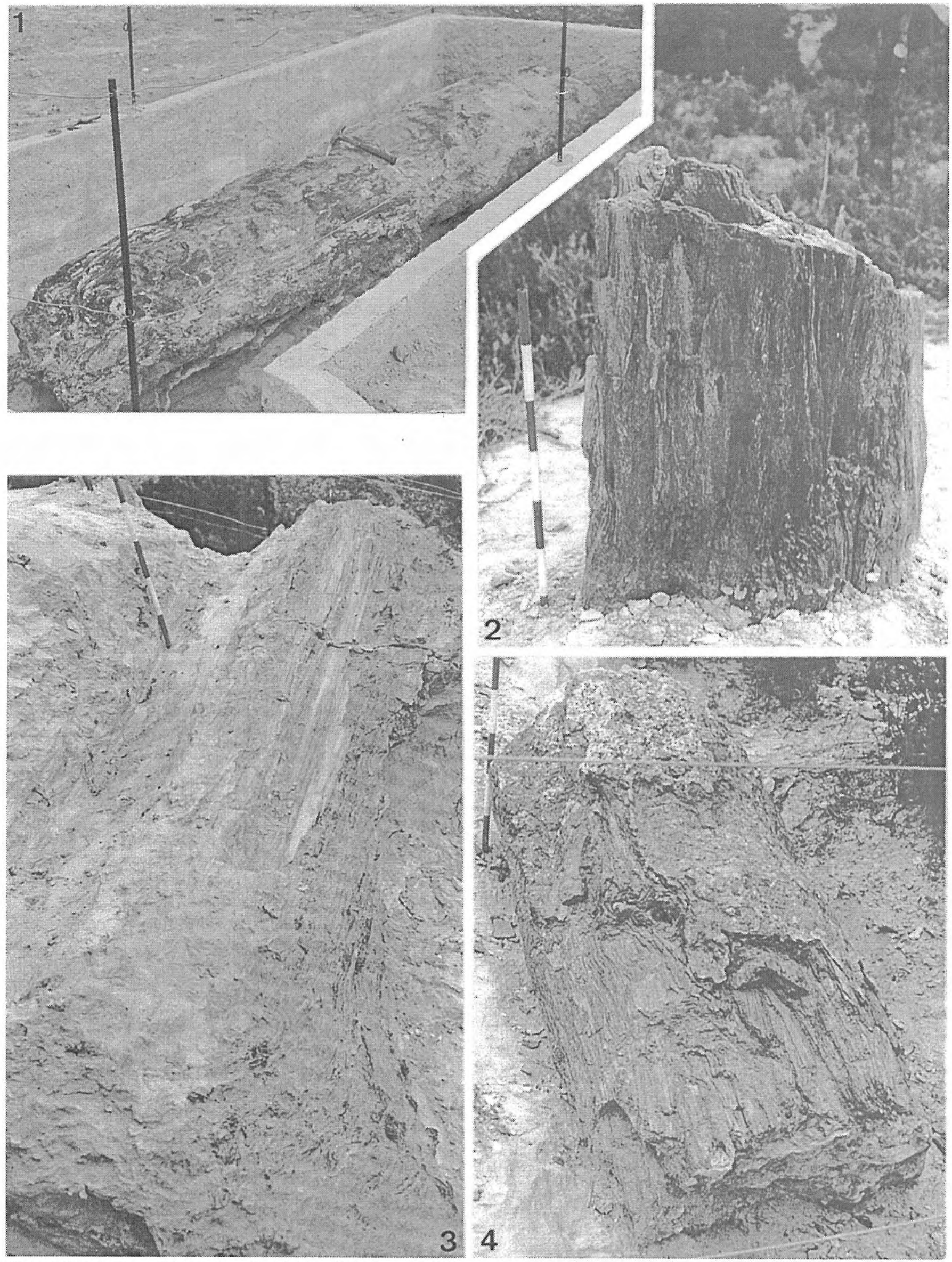

Revista Española de Paleontología, 14 (2), 1999. 


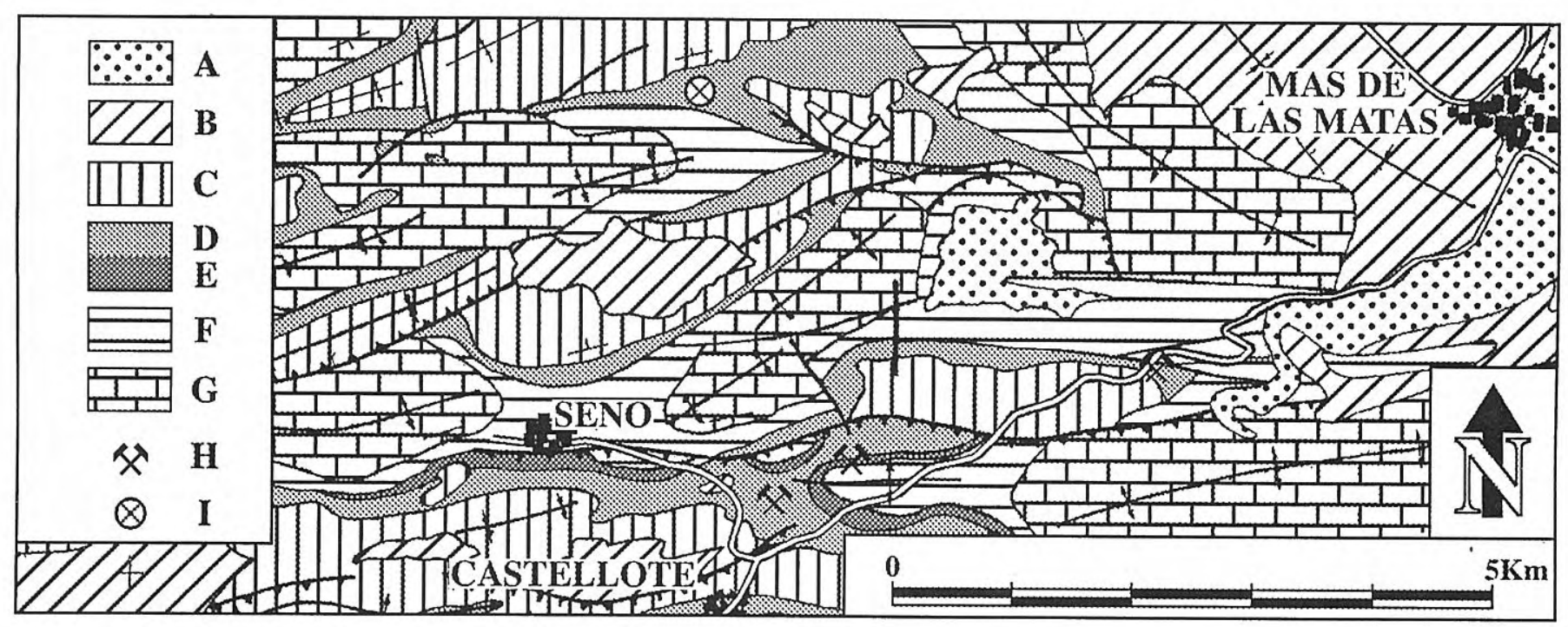

Figura 2. Encuadre geológico del yacimiento. Leyenda: A.- Cuaternario, B.- Paleoceno-Plioceno, C.- CenomaniensePaleoceno, D.- Formación Utrillas y E.- Formación Escucha, Aptiense sup.-Albiense, F.- Hauteriviense-Aptiense superior, G.- Jurásico, H.- Mina e I.- yacimiento.

presenta potentes sets de estratificación cruzada en surco, y un término lutítico de potencia decamétrica, coincide con la secuencia tipo U-3, identificada por estos autores en la parte inferior y media de la formación en el sector del Maestrazgo. Se propone para esta secuencia una génesis integramente fluvial, caracterizada por estructuras y geometrías propias de este ambiente.

La Formación Utrillas carece de dataciones propias, por lo que su atribución cronoestratigráfica se ha realizado a partir de la datación de las unidades litoestratigráficas que la suceden verticalmente en continuidad sedimentaria. La base de la Fm. Mosqueruela, suprayacente a la Fm. Utrillas y en continuidad sedimentaria con la misma, contiene orbitolínidos del Albiense superior (Pardo et al., 1991), por lo que en este sector la Formación Utrillas representa sólo una parte del Albiense superior.

\section{DESCRIPCIÓN DEL YACIMIENTO Y DEL MATERIAL ESTUDIADO}

La extensión total del yacimiento de "El Barranquillo" es desconocida ya que sólo se ha excavado la sección de la Formación Utrillas donde afloraban en superficie los troncos fósiles. Destacan cuatro de los troncos por su buena conservación y sus grandes dimensiones. Estos ejemplares, aún sin estar completos, presentan longitudes entre $1,5 \mathrm{~m}$ y 4,75 m, con diámetros entre 0,5 y 0,7 m (Lám. I, figs. 1, 2, 3 y 4). Tres de ellos conservan su posición original de depósito, paralelos entre sí y tendiendo a ser perpendiculares con la dirección de la corriente del paleocanal. La dirección media de los troncos, sin haberse realizado correcciones por el buzamiento de los estratos al ser prácticamente nulo, es 088, mientras que la dirección de la corriente, deducida a partir de la dirección de las láminas de estratificación cruzada, es de 150, existiendo entre ambas una diferencia de $62^{\circ}$. La situación del tronco restante se ha modificado a causa de la erosión. Entre estos grandes restos aparecen multitud de fragmentos más pequeños de longitud variable (desde $5 \mathrm{~cm}$ a $40 \mathrm{~cm}$ ), derivados de la erosión de los ejemplares mayores.

Todos los fragmentos están constituidos únicamente por xilema, que muchas veces se presenta desgajado paralelamente al eje del tronco (Lám. I, fig. 3). No se observan restos de médula, floema ni corteza, y en los grandes ejemplares tampoco aparecen ramificaciones ni raíces.

Respecto al estado de conservación, todos los ejemplares aparecen como restos silicificados más o menos recristalizados. Los procesos de silicificación favorecen la conservación de la estructura celular, mientras que las recristalizaciones conllevan la destrucción de la misma, como se explica en el apartado de tafonomía. Por esta razón, se han escogido para llevar a cabo el estudio tafonómico aquellas muestras en las que menos han actuado los procesos de recristalización.

El estudio preliminar de los ejemplares revela que todos los fragmentos de xilema recogidos pertenecen al grupo de las Gimnospermas. Todos ellos, así como las láminas delgadas estudidas, se encuentran depositados en el Museo Paleontológico de la Universidad de Zaragoza (MPZ 97/2504 a MPZ 97/2522).

\section{TAFONOMÍA. DESCRIPCIÓN DE LOS PROCESOS RECONOCIDOS}

Los procesos tafonómicos que afectan a los restos vegetales pueden dividirse en tres categorías, atendiendo a su naturaleza y a su sucesión temporal (Gastaldo, 1988). Todos estos procesos han sido identificados por Diez et al. (1996) en el yacimiento de troncos fósiles de "El Barranquillo".

Los primeros en actuar son los procesos necrológicos, que se suceden desde la muerte del ejemplar hasta el comienzo de la actuación de los agentes sedimentarios. A partir de aquí tienen lugar los procesos bioestratinómicos, 
interpretados como la interacción entre los restos vegetales y el medio sedimentario. Por último, se reconocen los procesos fosildiagenéticos, es decir, aquellos que se producen desde el enterramiento de los ejemplares hasta su hallazgo.

\section{PROCESOS NECROLÓGICOS}

\section{Muerte}

En los troncos que aparecen en el yacimiento de "El Barranquillo" únicamente se distinguen elementos propios del xilema, faltando los tejidos medulares, floemáticos y corticales. Puesto que estos tejidos son los primeros en desaparecer tras la muerte de un árbol, se deduce que los ejemplares estudiados estaban muertos antes de ser arrancados. Se explica así la relativa facilidad con la que la corriente pudo separar los troncos de las raíces (también ausentes en los ejemplares del yacimiento), al tratarse de elementos huecos en avanzado estado de descomposición. Evidentemente, los troncos ofrecieron resistencia, observándose en los ejemplares desgajamientos y desgarres del xilema paralelos al eje del tronco, pero la energía requerida para llevar a cabo el proceso podría conseguirse durante una avenida del río. Esta avenida no estaba relacionada con procesos estacionales, como demuestra la ausencia de anillos de crecimiento en el xilema de los ejemplares (Lám. II, figs. 5 y 6 ) debida a la constancia de las condiciones climáticas, con pocas variaciones a lo largo del año.

\section{PROCESOS BIOESTRATINÓMICOS}

\section{Transporte y emplazamiento}

La corriente integró al cauce fluvial los troncos secos y huecos cercanos a los márgenes del río, fracturándolos por encima de sus bases. Junto con estos restos también pudieron ser arrastrados troncos caídos, ramas y otros fragmentos de vegetación que, debido a su morfología y dimensiones, sufrieron diferentes procesos de transporte y emplazamiento.

Aunque no se puede cuantificar la magnitud del transporte sufrido, que continuó hasta que la corriente no tuvo energía suficiente para transportar los ejemplares, la gran uniformidad de tamaño que presentan los troncos estudiados en "El Barranquillo" señala un transporte lo suficientemente largo para que se haya podido producir la selección.

Cuando la longitud de un objeto flotante es mayor que su anchura, como sucede en los ejemplares estudiados, el eje longitudinal se alinea paralelo a la dirección de la corriente. La base de los troncos (más pesada) se situa a favor de la misma. Cuando la energía del medio no es la suficiente para continuar transportándolos, los ejemplares se encallan por su zona más ancha y pesada, y la corriente tiende a girarlos. Este giro continúa hasta que el ángulo que forman respecto a la fuerza de empuje provoca que el tronco, antes que seguir girando, tienda a desplazarse rodando. De esta forma los troncos de "El Barranquillo" alcanzan su emplazamiento definitivo formando un ángulo medio de $62^{\circ}$ con la dirección de transporte del cauce fluvial. Esta orientación principal respecto al sentido de la corriente permite desechar que se haya producido una reelaboración de los ejemplares.

\section{Enterramiento}

El fenómeno de enterramiento se produjo por efecto de la propia sedimentación fluvial, como consecuencia de la continua acumulación de los materiales detríticos que el cauce transportaba. Puede asegurarse que este enterramiento fue relativamente rápido, favoreciendo así el posterior desarrollo de los procesos de carbonificación, bajo condiciones de anoxia. El mecanismo descrito permite desechar que haya tenido lugar una reelaboración de los ejemplares, que habría requerido un improbable incremento de energía de considerable magnitud en el medio. Esta observación, junto con la expuesta en el apartado anterior, permiten proponer que los ejemplares son entidades resedimentadas.

Conforme el proceso de enterramiento avanza en el tiempo, la carga litostática aumenta sobre los ejemplares. Comienzan entonces a actuar los fenómenos de compactación que continúan durante la fosildiagénesis. Sin embargo, no se aprecian deformaciones importantes en los troncos que afecten notablemente a la estructura. Son tres los factores principales que minimizan los efectos de la carga: la consistencia propia de los tejidos leñosos que intervienen en el proceso, la temprana permineralización de los troncos y la plasticidad del sedimento que los englobaba, que tiende a adaptarse a la forma de los ejemplares antes que a deformarlos.

\section{PROCESOS FOSILDIAGENÉTICOS}

Estos procesos se han deducido a partir de las observaciones realizadas al microscopio petrográfico sobre diferentes láminas delgadas pertenecientes a distintos fragmentos de troncos que se recogieron en el yacimiento. Se han diferenciado tres etapas:

\section{Procesos de carbonificación}

Este proceso se inicia durante la diagénesis temprana y continúa hasta alcanzar un grado medio definido por la presencia de macerales del grupo vitrinita e inertinita (Stach et al., 1982). El proceso de carbonificación llega a ser representativo, observándose en algunas muestras porcentajes del $25 \%$ en restos carbonosos (Lám. Il, fig. 1). Para que este proceso tenga lugar es necesario que el enterramiento de los troncos se produzca rápidamente y bajo condiciones anóxicas. La liberación de $\mathrm{CO}_{2}$ al medio durante la carbonificación genera microambientes ácidos que favorecen el proceso físico-químico de fijación de la sílice.

\section{Procesos de silicificación}

Son contemporáneos e interdependientes de los procesos de carbonificación. La silicificación de las muestras es parcial y se produce tanto por los procesos físicos-químicos anteriormente mencionados como por los procesos bioquímicos determinados por la unión por puentes de hidrógeno que se forman entre los grupos 
hidroxilo del ácido silícico y los radicales libres de la materia orgánica. No se trata, por lo tanto, de un proceso de reemplazamiento, sino de una permineralización que replica al detalle las características histológicas de los ejemplares.

Se distinguen dos fases en este proceso:

a.- Fijación de sílice en los tabiques celulares (Lám. II, fig. 3), originalmente compuestos de lignina o celulosa, que da lugar a estructuras zonadas y al reemplazamiento parcial por variedades fibrosas de la sílice, tales como la calcedonita. Esta permineralización es la responsable de que se conserven las características histológicas de las paredes de los vasos leñosos.

b.- La saturación en sílice del medio baja, produciéndose el relleno de las cavidades celulares (Lám. II, fig. 3). La silicificación en este caso es más lenta que en el anterior, favoreciendo la formación de cuarzo monocristalino en los huecos de las cavidades celulares. Este proceso establece las diferencias texturales entre pared y lumen celular, facilitando su diferenciación.

Esta silicificación diferencial favorece la conservación en los ejemplares de las características celulares del xilema, como pueden ser la estructura de los radios medulares (Lám. II, fig. 6) o la presencia de canales secretores traumáticos (Lám. II, fig. 5), posibilitando el estudio histológico de las muestras.

Un problema añadido al estudio de la silicificación es deducir el origen de la sílice que transportan las soluciones del sistema. Se descartan, como fuentes posibles, la desvitrificación de cuarzos volcánicos (no existen evidencias cercanas de vulcanismo), la disolución por presión de los cuarzos y el aporte a partir de la agradación de los minerales arcillosos presentes (durante la diagénesis media no se dan las condiciones de presión y temperatura necesarias para que se produzcan estos procesos), el origen biogénico de la sílice (a partir de las caracterísiticas litológicas de la unidad en la que aparecen los fósiles y de los sedimentos supra e infrayacentes), y la precipitación de fases coloidales de la misma (más característica de medios alcalinos). Estas afirmaciones se apoyan también en el estudio petrográfico de láminas delgadas de las muestras estudiadas, en las que no se observan evidencias texturales de ninguno de estos procesos.

Las areniscas de la Formación Utrillas, sin embargo, presentan en la matriz caolinita como componente primario, en concentraciones que en ocasiones llegan a ser explotables, como ocurre en las inmediaciones del yacimiento de "El Barranquillo". Se plantea, por lo tanto, como fuente de sílice más probable la alteración de los feldespatos potásicos, que formaban parte del sedimento inicial, a caolinita, que conlleva la liberación de sílice al medio en forma de ácido silícico.

\section{Procesos de recristalización}

Son procesos posteriores a la carbonificación y la silicificación. En las muestras estudiadas comienzan durante la diagénesis media, observándose dos procesos de recristalización que se suceden en el tiempo:

- Formación de cuarzo monocristalino en los tabiques celulares a partir de las morfologías fibrosas preexistentes.

- Desarrollo de cristales hiperblásticos (Jefferson, 1983) a partir de los cuarzos de relleno de cavidades celulares y de los de recristalización de los tabiques.

Esta última transformación, consecuencia de la baja saturación del medio en sílice, es la responsable de la destrucción de los detalles más delicados de la estructura de la madera, al crecer los cristales a través de las paredes celulares.

En función de la permeabilidad del sistema estos procesos de recristalización generan estructuras diferenciales:

-En las zonas asociadas a fracturas (Lám. II, figs. 2 y 4) se manifiestan como estructuras zonadas con un núcleo central en las que se aprecian áreas con cuarzos hiperblásticos rodeados de una envuelta externa con cuarzos petaloides, consecuencia de la recristalización diferencial.

-En las zonas masivas (no afectadas por fracturas) (Lám. II, figs. 2 y 3), este proceso da lugar a recristalizaciones en "mosaico grueso" (Diez et al., 1996), caracterizadas por cristales de cuarzo hiperblásticos que presentan morfologías idioblásticas hacia las superficies libres.

\section{Lámina II}

Vistas al microscopio petrográfico de cortes transversales de troncos silicificados pertenecientes al yacimiento de "El Barranquillo”. Imágenes tomadas con nícoles paralelos, excepto la figura 2, tomada con nícoles cruzados.

1 Ejemplar MPZ 97/2522 con porcentajes de carbonificación próximos al $25 \%$.

2 Ejemplar MPZ 97/2519 presentando la textura en "mosaico grueso", en el que también se observa el desarrollo de estructuras petaloides (a).

3 Ejemplar MPZ 97/2521 con procesos de recristalización asociados a zonas masivas. Se pueden distinguir los procesos de silicificación de relleno de cavidades celulares (a) y permineralización de tabiques (b), así como el desarrollo durante la recristalización de cristales de cuarzo idioblásticos (c) aprovechando las superficies libres.
4 Ejemplar MPZ 97/2520 con procesos de recristalización asociados a zonas de fracturas. Pueden diferenciarse claramente las zonas centrales con cuarzos hiperblásticos (a) de las envueltas externas con cuarzos petaloides (b).

5 Ejemplar MPZ 97/2517 en el que se observa la conservación de la estructura celular, llegándose a apreciar la presencia de canales secretores traumáticos (a).

6 Ejemplar MPZ 97/2514 en el que se observa la conservación de las estructuras celulares de las traqueidas y radios medulares. 
Lámina II

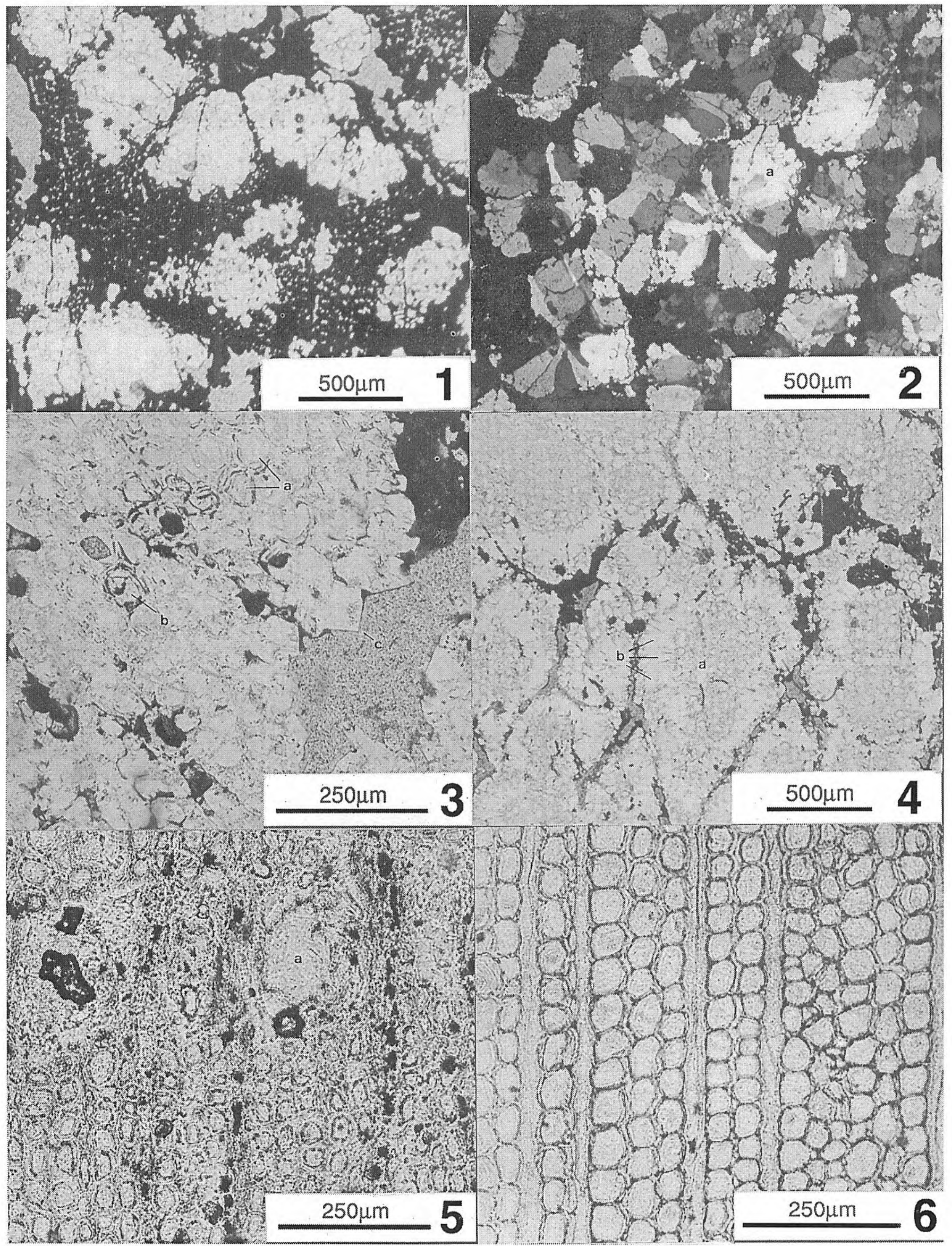

Revista Española de Paleontología, 14 (2), 1999. 


\section{CONCLUSIONES}

La gran calidad de afloramiento del yacimiento de troncos fósiles de "El Barranquillo", de edad Albiense, ha permitido estudiar de manera clara su historia y evolución.

El arranque de estos troncos, provocado por una avenida no estacional, supuso la integración al cauce fluvial de individuos ya muertos que formaban parte de un bosque con predominio de coníferas. El transporte de estos troncos fue considerable, viéndose favorecido por la total ausencia de ramas, raíces u otros elementos que pudiesen dificultar su descenso.

Tras su depósito, tuvieron lugar los procesos de carbonificación que implican un enterramiento rápido en medio anóxico. Este proceso, que se prolongó hasta la diagénesis media, generó microambientes ácidos que favorecieron los procesos de silicificación responsables de la conservación de las características celulares de los ejemplares. Posteriormente, con el aumento de la presión y la temperatura, tuvieron lugar los procesos de recristalización de la sílice en dos fases (formación de cuarzo monocristalino y desarrollo de cristales hiperblásticos), dando lugar a estructuras diferenciales.

\section{AGRADECIMIENTOS}

Este trabajo ha sido subvencionado por el proyecto CONAI PCB 06/93, el programa Europa-CAI, el Instituto de Estudios Turolenses, el Museo de Mas de las Matas y el Grupo de Estudios Masinos.

Este trabajo es una contribución al proyecto de la D.G.I.C.Y.T. PB 92-0862 C02-02.

Agradecemos a las doctoras Denise Pons y Colette Vozenin-Serra del Laboratorie de Paléobotanique et Paléoécologie de l'Université Pierre et Marie Curie de Paris su enseñanzas e indicaciones.

Igualmente queremos agradecer a los doctores Juan Mandado, Gonzalo Pardo y Alfonso Meléndez, de las áreas de Petrología y Estratigrafía del Departamento de Ciencias de la Tierra de la Universidad de Zaragoza, la revisión desinteresada de los diferentes datos presentados en este trabajo.

Agradecemos también al Dr. Sixto Fernández López, del Departamento de Paleontología de la Universidad Complutense de Madrid, sus oportunas sugerencias y observaciones y a la Dra. Carmen Diéguez, del Museo Nacional de Ciencias Naturales, la lectura crítica del manuscrito.

\section{BIBLIOGRAFÍA}

Aguilar, M. J., Ramírez del Pozo, J. y Riba, O. 1971. Algunas precisiones sobre la sedimentación y paleoecología del Cretácico Inferior en la zona UtrillasVillarroya de los Pinares. Estudios Geológicos, 27, $497-$ 512.

Canerot, J. 1974. Recherches géologiques aux confins des Chaînes ibérique et catalan (Espagne). These Doctorelle Sciences Naturelles Toulouse. ENADIMSA, Trabajos de tesis, serie $5, n^{\circ} 4,517$ pp.

Diez, J. B., Canudo, J. I., Ferrer, J., Muñoz Barragán, P., Ruiz-Omeñaca, J. I. y Soria, A. R. 1996. Transporte y resedimentación de troncos silicificados en el Albiense (Fm. Utrillas, Castellote, Cordillera Ibérica). In: II Reunión de Tafonomía y fosilización (Eds. G. Meléndez, M. F. Blasco e I. Urresti). Institución "Fernando el Católico", Zaragoza, 97-102.

Gastaldo, R. A. 1988. A conspectus of Phytotaphonomy. In: Methods and Applications of Plant Paleoecology. (Eds. W. A. DiMichele and S. L. Wing) The Paleontological Association Special Publication, 3, 14-28.

Guimerà, J. 1983. Evolution de la deformation alpine dans le NE de la Chaîne Ibérique et dans la Chaîne Cotiere Catalane. Comptes Rendus de l'Académie des Sciences, Série 111, 297, 425-430.

Guimerà, J. 1988. Estudi estructural de l'enllaç entre la Serralada Ibérica i la Serralada Costanera Catalana. Tesis Doctoral, Universidad Barcelona, 600 pp.

Jefferson, T. H. 1983. The preservation of conifer wood: Examples from the Lower Cretaceous of Antarctica. Palaeontology, 30, 233-249.

Lemoigne, Y. et Marin, P. 1972. Bois silicifiés du Cretacé Inférieur continental de la province de Teruel (Espagne). Extrait du Bulletin Mensuel de la Société Linnéenne de Lyon, 10, 201-214.

Pardo, G. 1979. Estratigrafía y sedimentología de las formaciones detríticas del Cretácico Inferior terminal en el Bajo Aragón Turolense. Tesis Doctoral. Universidad de Zaragoza. 2 tomos. (Inédita).

Pardo, G., Arderol, L. y Villena, J. 1991. Sedimentología de las Fms. Escucha y Utrillas. In: Memoria del Mapa Geológico de España, Hoja de Daroca $\left(n^{\circ} 40\right)$. E. 1:200.000, ITGE, Madrid, 239 pp.

Stach, E., Mackowsky, M. Th., Teichmuller, M., Taylor, G. H., Chandra, D. and Teichmuller, R. 1982. Textbook of Coal Petrology. Gebruder Borntraeger, Stuttgart, 428 pp.

Manuscrito recibido: 2 de junio, 1997 Manuscrito aceptado: 14 de abril, 1999 